\title{
A Non-rigid Registration Method for Serial microCT Mouse Hindlimb Images
}

\author{
Jung W. Suh ${ }^{1}$, Dustin Scheinost ${ }^{1}$, Donald P. Dione ${ }^{2}$, Lawrence W. Dobrucki ${ }^{2}$, \\ Albert J. Sinusas ${ }^{1,2}$, and Xenophon Papademetris ${ }^{1,3}$ \\ 1 Department of Diagnostic Radiology, Yale School of Medicine, New Haven, CT \\ 2 Department of Internal Medicine, Yale School of Medicine, New Haven, CT \\ 3 Department of Biomedical Engineering, Yale University New Haven, CT
}

\begin{abstract}
We present a new method for the non-rigid registration of serial mouse microCT images which undergo potentially large changes in the positions of the legs due to articulation. While non-rigid registration methods have been extensively used in the evaluation of individual organs, application in whole body imaging has been limited, primarily because the scale of possible displacements and deformations is large resulting in poor convergence of most methods. Our method is based on the extended demons algorithm that uses a level-set representation of the mouse skin and skeleton as an input, and composed of three steps reflecting the natural physical movements of bony structures. We applied our method to the registration of serial microCT mouse images demonstrating encouraging performances as compared to competitive techniques.
\end{abstract}

\section{Introduction}

Whole body mouse registration is a challenging emerging problem in image analysis with many potential applications. These applications arise from the extensive genetic similarities between the mouse and human genomes which make the mouse an excellent model for understanding human developmental processes and pathologies. A particular mouse model of interest in our work is that of hindlimb ischemia [1, which is used to study vascular remodeling. In this model an ischemic zone is created in one limb by surgical ligation of the femoral artery, with the other limb serving as a paired control. Vascular remodeling is an important process which plays an important role in both peripheral arterial disease and coronary artery disease.

Molecular imaging using microSPECT can be used to quantify angiogenesis, the process of formation of new blood vessels. This is often performed using hybrid microSPECT/microCT scanners. A key challenging in the quantification of the SPECT images is the parcellation of the hindlimbs into different anatomical regions (e.g. proximal, distal and foot) to enable the region-of-interest analysis of these images. Whereas such parcellation can be done manually (using the anatomy from the co-aquired microCT), this is tedious and leads to subjective divisions. Our goal in this work is to develop atlas-based labeling of the hindlimbs where the parcellation is done manually at the first time point and automatically 
propagated in time using our non-rigid registration method which we describe in this paper.

Registering the hindlimbs is probably the most challenging aspect of whole body mouse registration. Previous approaches to the problem of whole body mouse registration 6 6/8] have used a combination of the robust point matching method (RPM) to first align the bone surface as an initial condition followed by an intensitybased registration which aims to improve accuracy in the soft-tissue structures. Our work differs from the previous work in the literature in that (i) we adopt an implicit surface registration method which relies on a distance-map parameterization of the bone and skin surfaces and (ii) in performing the surface registration we follow the natural "anatomical" constraints of first registering the bone surfaces - which literally anchor the rest of the body and then refining this by introducing skin surface information. The result of the surface-based registration is then augmented by an intensity based non-rigid registration as before. We validate our results using 14 image-pairs using both intensity similarity criteria and measures of (implicit) segmentation accuracy as evaluated using standard overlap measures.

We compare our results to standard methods such as the demons method (which uses the same transformation model and optimization method as our method), the intensity-based non-rigid registration with free-form deformation (IFFD) 11] and a robust point-based method (RPM) 9] - which forms the basis of most of the previous mouse non-rigid registration work in the literature.

\section{Methods}

Figure 1 shows the brief block diagram of the proposed method. The inputs are microCT images of the same mouse imaged over a period of 4 weeks. This method is a three-step serial registration composed of a bone surface registration, a skin surface registration and an intensity registration. After initially executing the bone surface registration $\left(f_{1}\right)$, we execute the skin surface registration $\left(f_{2}\right)$ based on the initial bone surface registration result. Then, we refine the image intensities for the soft tissue between the bone and skin by the final intensity registration $\left(f_{3}\right)$. We combine these three registrations as one registration using concatenation $\left(f_{\text {total }}=f_{3} \circ f_{2} \circ f_{1}\right)$. We first review an extended demons method for single surface mapping such as a bone surface. Then we describe each step in the proposed method.

\subsection{Extended Demons Registration}

Thirion 2] suggested an image-matching algorithm using a diffusion process. Using the demons concept from Maxwell's demons in thermodynamics, the nonrigid image matching process is modeled as a diffusion process in the demons algorithm. The theory behind this algorithm is that the object boundary can change its shape depending on the position of the demons within the image domain. The demons' forces, which deform the object shape, are generated to reduce the disparity between the reference and the moving images. Equation (1) 


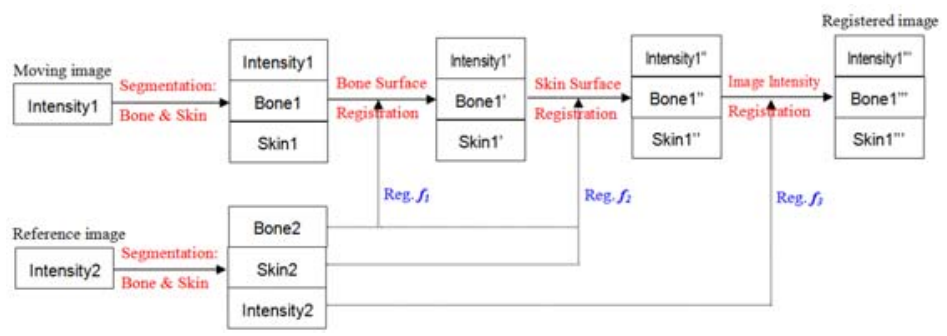

Fig. 1. Block diagram for our registration method

shows the demons algorithm based on the optical flow equation. Here, $\mathbf{r}(\mathbf{X})$ is the reference image, $\mathbf{m}(\mathbf{X})$ is the moving image and $\mathbf{v}(\mathbf{X})$ is the displacement.

$$
\mathbf{v}(\mathbf{X})=-((\mathbf{m}(\mathbf{X})-\mathbf{r}(\mathbf{X})) \nabla \mathbf{r}(\mathbf{X})) /\left(\|\nabla \mathbf{r}(\mathbf{X})\|^{2}+(\mathbf{m}(\mathbf{X})-\mathbf{r}(\mathbf{X}))^{2}\right)
$$

Many studies improved the original demons algorithm by increasing the speed of convergence 45. For stronger convergence performance, the extended demons registration method takes advantage of the level-set representation of the object boundary using a distance map based on the demons algorithm. The extended demons algorithm utilizes the distance map around the shape border instead of intensity which results in improved convergence even though the two objects are not overlapped sufficiently. Thus, the extended demons registration algorithm can achieve much higher convergence speed with better confidence in binary volume registration. In this paper, we used a symmetric version of the extended demons method to avoid local minima as below.

$$
\mathbf{v}(\mathbf{m}, \mathbf{r}, \mathbf{X})=-\frac{2(\mathbf{m}(\mathbf{X})-\mathbf{r}(\mathbf{X}))(\nabla \mathbf{r}(\mathbf{X})+\nabla \mathbf{m}(\mathbf{X}))}{\|\nabla \mathbf{r}(\mathbf{X})+\nabla \mathbf{m}(\mathbf{X})\|^{2}+(\mathbf{m}(\mathbf{X})-\mathbf{r}(\mathbf{X}))^{2}}
$$

\subsection{First Step: Registration of Bone Surface}

From each reference and moving CT images of the same mouse, binary bone images and binary skin images are segmented through intensity differences by thresholding and clustering (K-means clustering with 3 classes)(Figure 1). The level-set represented bone images are generated by distance map from the binary reference bone image and the binary moving bone image respectively. Using $\mathbf{v}\left(\mathbf{m}_{B}, \mathbf{r}_{B}, \mathbf{X}\right)$ of the extended demons algorithm (equation (2) ), the deformation between the two level-set represented bone images is calculated.

Here, $\mathbf{r}_{B}$ is the reference image represented by level-set from the bone boundary, $\mathbf{m}_{B}$ is the moving image represented by level-set from the bone boundary, and $\mathbf{v}\left(\mathbf{m}_{B}, \mathbf{r}_{B}, \mathbf{X}\right)$ is the displacement between $\mathbf{r}_{B}$ and $\mathbf{m}_{B}$.

\subsection{Second Step: Registration of Skin Surface}

After the registration of bone surface in the first step, the moving skin image is deformed with the displacement of the bone surface registration result. In the same way as the bone registration, the level-set represented skin images 
are generated by distance maps from the binary reference skin image and the binary moving skin image respectively. Using $\mathbf{v}\left(\mathbf{m}_{1 S}, \mathbf{r}_{S}, \mathbf{X}\right)$, we calcualte the deformation between the two level-set represented skin images. Here, $\mathbf{r}_{S}$ is the reference image represented by level-set from the skin boundary, $\mathbf{m}_{1 S}$ is the moving image represented by level-set from the skin boundary (deformed by the result $\left(\mathbf{v}\left(\mathbf{m}_{B}, \mathbf{r}_{B}, \mathbf{X}\right)\right)$ of the first step), and $\mathbf{v}\left(\mathbf{m}_{1 S}, \mathbf{r}_{S}, \mathbf{X}\right)$ is the displacement between $\mathbf{r}_{S}$ and $\mathbf{m}_{1 S}$. In this second step, bone surfaces are registered again simultaneous (during a same iteration) with the skin surface registration using $\mathbf{v}\left(\mathbf{m}_{1 B}, \mathbf{r}_{B}, \mathbf{X}\right)$. Here, $\mathbf{m}_{1 B}$ is the moving image represented by level-set from the bone boundary (deformed by the result $\left(\mathbf{v}\left(\mathbf{m}_{B}, \mathbf{r}_{B}, \mathbf{X}\right)\right)$ of the first step), and $\mathbf{v}\left(\mathbf{m}_{1 B}, \mathbf{r}_{B}, \mathbf{X}\right)$ is the displacement between $\mathbf{r}_{B}$ and $\mathbf{m}_{1 B}$.

During each iteration of the extended demons algorithm the two displacements $\left(\mathbf{v}\left(\mathbf{m}_{1 B}, \mathbf{r}_{B}, \mathbf{X}\right)\right.$ and $\left.\mathbf{v}\left(\mathbf{m}_{1 S}, \mathbf{r}_{S}, \mathbf{X}\right)\right)$ are spatially combined in every iteration by equation (3) within the $f_{2}$ in Figure 1.

$$
\mathbf{v}(\mathbf{X})=\mathbf{w}_{B}(\mathbf{X}) \mathbf{v}\left(\mathbf{m}_{1 B}, \mathbf{r}_{B}, \mathbf{X}\right)+\mathbf{w}_{S}(\mathbf{X}) \mathbf{v}\left(\mathbf{m}_{1 S}, \mathbf{r}_{S}, \mathbf{X}\right)
$$

Where $\mathbf{w}_{B}(\mathbf{X})$ and $\mathbf{w}_{S}(\mathbf{X})$ are weight factors defined as:

$$
\begin{gathered}
w_{B}\left(x_{k}\right)=\left\{\begin{array}{cl}
1 & \text { if } d_{B}\left(x_{k}\right) \leq 0 \text { and } d_{S}\left(x_{k}\right)<0 \\
0 & \text { if } d_{B}\left(x_{k}\right)>0 \text { and } d_{S}\left(x_{k}\right) \geq 0 \\
\frac{\left|d_{S}\left(x_{k}\right)\right|}{\left|d_{B}\left(x_{k}\right)\right|+\left|d_{S}\left(x_{k}\right)\right|} & \text { if } d_{B}\left(x_{k}\right)>0 \text { and } d_{S}\left(x_{k}\right)<0
\end{array}\right. \\
w_{S}\left(x_{k}\right)=\left\{\begin{array}{cl}
0 & \text { if } d_{B}\left(x_{k}\right) \leq 0 \text { and } d_{S}\left(x_{k}\right)<0 \\
1 & \text { if } d_{B}\left(x_{k}\right)>0 \text { and } d_{S}\left(x_{k}\right) \geq 0 \\
\frac{\left|d_{B}\left(x_{k}\right)\right|}{\left|d_{B}\left(x_{k}\right)\right|+\left|d_{S}\left(x_{k}\right)\right|} & \text { if } d_{B}\left(x_{k}\right)>0 \text { and } d_{S}\left(x_{k}\right)<0
\end{array}\right.
\end{gathered}
$$

Here $\left|d_{B}\left(x_{k}\right)\right|$ is the absolute value of the signed distance value and $k$ is the voxel index. The displacement of the bone registration $\mathbf{v}\left(\mathbf{m}_{1 B}, \mathbf{r}_{B}, \mathbf{X}\right)$ is exclusively used within the bone region, and the displacement of the skin registration $\mathbf{v}\left(\mathbf{m}_{1 S}, \mathbf{r}_{S}, \mathbf{X}\right)$ is exclusively used outside the skin region. The displacement of the soft tissue between bone and skin is calculated by interpolation depending on the distance from both the bone and skin. In order to reduce the abrupt change of the displacement, the standard deviation of the smoothing filter within the extended demons algorithm is set to a large value ( $\sigma=2$ voxels.)

\subsection{Third Step: Registration of Graylevel Intensity Image}

After finishing the registration of bone and skin surfaces, soft tissues between the bone and skin are registered through this third step. In the third step, the symmetric version of the demons algorithm [2] with histogram matching is used for the image intensity registration instead of the extended demons algorithm. The moving graylevel intensity image is deformed with both the displacement $\left(f_{1}\right)$ of the bone surface registration result and the displacement $\left(f_{2}\right)$ of the skin surface registration result as shown in Figure 1. The deformed moving graylevel intensity image and the reference graylevel intensity image are used as inputs of this third step of registration. 


\subsection{Implementation Details}

Three resolution layers were utilized in each step from the coarsest to the finest layer to search the displacement in the $3 \mathrm{D}$ data set. Iteration numbers for each layer were set to 200, 200 and 30 from the coarsest to the finest layer, respectively.

The images consisted of the lower half of the mouse (roughly from below the lungs to the feet). The image size after cropping was approximately $160 \times 90 \times$ 140 (voxels).

\section{Results}

We present results of the application of our method to $N=14$ pairs of mouse microCT images, acquired with a resolution of $100 \times 100 \times 100 \mu^{3}$. In these images the mice are virtually divided into 7 regions shown in Figure 2 . These enables the comparison of the overlap of these regions after registration. In order to generate this mouse parcellation, we manually positioned planes to segment the

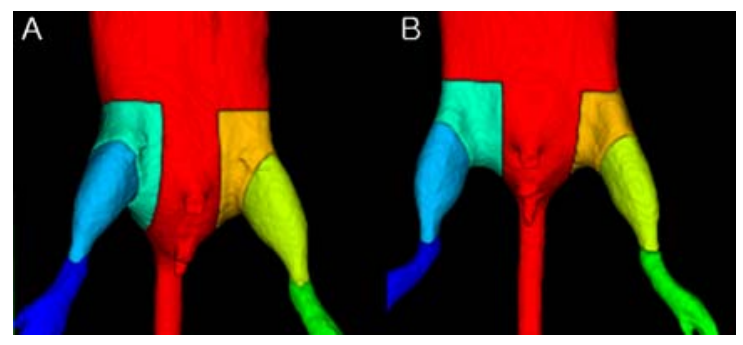

Fig. 2. 7-region mouse parcellation; body and tail (red), the left proximal hindlimb (yellow), the left distal hindlimb (light green), the left foot (green), the right proximal (light teal), the right distal (light blue), and the right foot (blue) (a) Baseline, (b) Moving

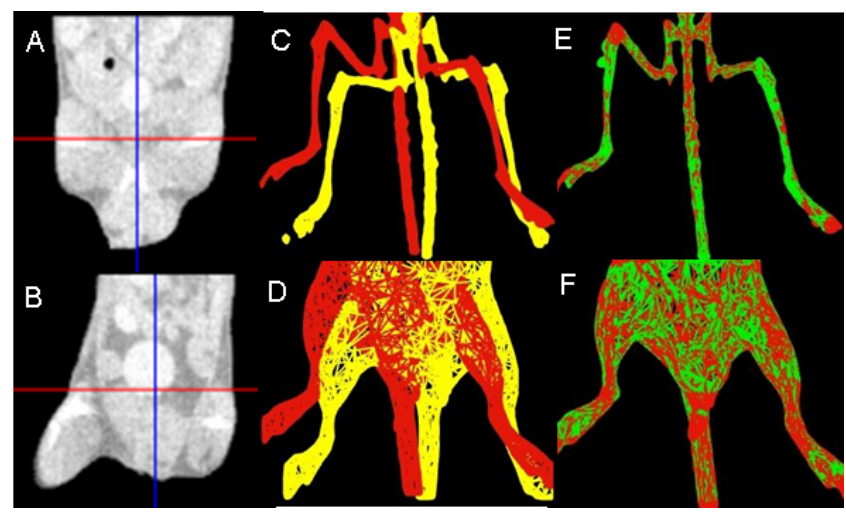

Fig. 3. (A) and (B) Reference and Targed Images. Bone (C) and Skin (D) Surfaces prior to Registration. Bone (E) and Skin (F) Surfaces after registration. 
Table 1. Similarity comparisons (Jaccard Similarity Coefficient) for the regions

\begin{tabular}{|c||c|c|}
\hline Right proximal & $J S C$ Averg. & T test \\
\hline \hline First step & 67.66 & $1.65 \mathrm{E}-04$ \\
\hline Second step & 78.36 & 0.66 \\
\hline $\begin{array}{l}\text { Third step } \\
\text { (Final reg) }\end{array}$ & $\mathbf{7 8 . 9 2}$ & \\
\hline IFFD & 76.50 & 0.30 \\
\hline RPM & 73.14 & 0.02 \\
\hline Demons & 64.67 & $1.10 \mathrm{E}-02$ \\
\hline
\end{tabular}

\begin{tabular}{|c||c|c|}
\hline Left proximal & $J S C$ Averg. & T test \\
\hline \hline First step & 65.31 & $3.9 \mathrm{E}-06$ \\
\hline Second step & 75.135 & 0.44 \\
\hline $\begin{array}{l}\text { Third step } \\
\text { (Final reg) }\end{array}$ & $\mathbf{7 5 . 7 0}$ & \\
\hline IFFD & 72.56 & $6.65 \mathrm{E}-05$ \\
\hline RPM & 74.26 & 0.29 \\
\hline Demons & 67.71 & $1.53 \mathrm{E}-02$ \\
\hline
\end{tabular}

\begin{tabular}{|c||c|c|}
\hline Right distal & $J S C$ Averg. & T test \\
\hline \hline First step & 63.44 & $2.03 \mathrm{E}-05$ \\
\hline Second step & 82.60 & 0.19 \\
\hline $\begin{array}{l}\text { Third step } \\
\text { (Final reg) }\end{array}$ & $\mathbf{8 5 . 2 6}$ & \\
\hline IFFD & 85.46 & 0.85 \\
\hline RPM & 79.49 & $9.08 \mathrm{E}-04$ \\
\hline Demons & 53.96 & $1.73 \mathrm{E}-03$ \\
\hline
\end{tabular}

\begin{tabular}{|c||c|c|}
\hline Left distal & $J S C$ Averg. & T test \\
\hline \hline First step & 62.40 & $9.76 \mathrm{E}-07$ \\
\hline Second step & 79.45 & 0.31 \\
\hline $\begin{array}{l}\text { Third step } \\
\text { (Final reg) }\end{array}$ & $\mathbf{8 0 . 4 2}$ & \\
\hline IFFD & 76.79 & $5.03 \mathrm{E}-02$ \\
\hline RPM & 70.78 & 0.029 \\
\hline Demons & 59.52 & $1.70 \mathrm{E}-03$ \\
\hline
\end{tabular}

\begin{tabular}{|c||c|c|}
\hline Right foot & $J S C$ Averg. & T test \\
\hline \hline First step & 60.53 & $4.61 \mathrm{E}-04$ \\
\hline Second step & 79.03 & 0.33 \\
\hline $\begin{array}{l}\text { Third step } \\
\text { (Final reg) }\end{array}$ & $\mathbf{8 3 . 2 9}$ & \\
\hline IFFD & 60.51 & $3.09 \mathrm{E}-04$ \\
\hline RPM & 58.77 & $6.53 \mathrm{E}-08$ \\
\hline Demons & 34.34 & $8.39 \mathrm{E}-05$ \\
\hline
\end{tabular}

\begin{tabular}{|c||c|c|}
\hline Left foot & JSC Averg. & T test \\
\hline \hline First step & 64.92 & $5.21 \mathrm{E}-08$ \\
\hline Second step & 78.72 & 0.40 \\
\hline $\begin{array}{l}\text { Third step } \\
\text { (Final reg) }\end{array}$ & $\mathbf{7 7 . 5 0}$ & \\
\hline IFFD & 55.36 & $7.54 \mathrm{E}-06$ \\
\hline RPM & 47.81 & $1.45 \mathrm{E}-06$ \\
\hline Demons & 19.57 & $2.13 \mathrm{E}-06$ \\
\hline
\end{tabular}

mouse into 7 regions; main body including the tail, the left proximal hindlimb, the left distal hindlimb, the left foot, the right proximal hindlimb, the right distal hindlimb, and the right foot. The planes were positioned on a $3 \mathrm{D}$ rendering of the CT image. Among those 7 regions, the main part of the body including the tail, is excluded as its upper boundary is variable between images. We compared our method to two non-rigid registration methods ([119]) and to the demons algorithm. We demonstrate encouraging improvement in registration accuracy compared to these standard methods. As a similarity measure for local shape, we used the Jaccard similarity coefficient $(J S C=(|R \cap M| /|R \cup M|) \times 100)$. Where $M$ is the moving and $R$ is the reference object, and $|M|$ refers the number of members in the set $M$ as a cardinality.

Table 1 shows the comparison results with the Jaccard Similarity Coefficient $(J S C)$ for the six mouse regions. In the first four tables in Table 1, the average JSCs of our proposed method (Third step) are slightly better or similar (Right distal hindlimb) to the average $J S C$ s of IFFD, and always better than those of the Demons algorithm and RPM. In cases of the $J S C$ s for both the right foot and left foot, however, our proposed serial registration has much 
better than all methods (much less than 0.05 in paired-T test). We note that our method achieves consistently high overlaps over the all six mouse regions and the improvement is particularly noticeable in the most challenging part the feet. This is probably due to two reasons. The intensity based only methods (IFFD,demons) can not converge in these region due to the large displacements that can be present. While the imaging technologists attempt to place the mice in roughly the same orientation (the scans are acquired in weekly intervals) this is almost impossible in the case of the feet resulting in large displacements which are less easily estimated by intensity-based methods. In the case of RPM, the improvement is most likely caused by the fact that the number of points that can be used for RPM is finite (we used about 5000 points) as it requires the computation of a match matrix which even if sparse, still becomes computationally intractable. This results in less accurate surface representation of smaller structures which explains partially why our method becomes increasingly superior to RPM as we move down the limb. (Please note that the surface only part of our algorithm - Second Step - also gives superior results to RPM, a surface-only technique, which is a more fair comparison.)

\section{Discussion and Conclusions}

We presented a new non-rigid stepped registration method for quantitiative evaluation of serial mouse images. Our method, which is based on an extended demons formulation, first aligns the bone surfaces to achieve a good initial condition and then leverages additional information obtained from skin surfaces to improve the result and finally includes the use of direct intensity information constrained by the pre-computed surface-derived transformations maps. These first and second steps of surface mappings are critical in ensuring that the intensity information is useful, as without a good initialization, intensity only methods fail to converge to the appropriate result. We evaluated the proposed method on $3 \mathrm{D}$ volumes from 14 serial mouse microCT data sets, and demonstrate that our method performs better three standard methods (IFFD, Demons and RPM.) The first two methods represent popular standard nonrigid intensity based registration algorithms which are readily available - and hence should be tried on a new problem prior to developing a customized method. The comparison to RPM was done as this has been used previously for whole body mouse registration.

Relating the comparison with Li et al. [7/8, this method is also a two-step algorithm. Step one is bone registration using robust point matching (RPM) from bone surface; step two is intensity-based registration. Although we don't use the same intensity-based registration method in the final step as Li et al., the intensity-based registrations in both cases are a "finishing" step. While we were not able to compare our results directly to the work of Li et al., we note that the surface only part of this algorithm (i.e. Step 2) demonstrates superior performance to the surface only portion of that method (which relied on RPM) for both sets of criteria. This is a strong indication that all things being equal our combined method is likely to perform better than this competitor in the challenging area of the hindlimbs. 
Our methodology may also be applicable to serial imaging of patients with peripheral arterial disease. In addition this type of multistage registration may be applicable to registration problems in the abdomen where there is the potential for large displacements of key structures such that conventional intensity-based methods are not able to handle. However, we emphasize that in much of this work, the goal is not to use the mouse as a test-bed for the development of imaging/image analysis methods which will eventually be translated to human applications. The goal is, rather, to develop mouse specific methods that will help understand the underlying pathology. It is this increased understanding that will ultimately most likely prove useful in a translational sense, as opposed to the methods themselves.

Acknowledgments. This work was supported in part by the NIH under grants R01HL065662 (AJS) and R01EB006494 (XP).

\section{References}

1. Nikol, S., Huehns, T.Y.: Preclinical and clinical experience in vascular gene therapy: advantages over conservative/standard therapy. Journal of Invasive Cardiology 13(4), 333-338 (2001)

2. Thirion, J.P.: Image matching as a diffusion process: an analogy with Maxwell's demons. Medical Image Analysis 2(3), 243-260 (1998)

3. Papademetris, X., Dione, D.P., Dobrucki, L.W., Staib, L.H., Sinusas, A.J.: Articulated rigid registration for serial lower-limb mouse imaging. In: Duncan, J.S., Gerig, G. (eds.) MICCAI 2005. LNCS, vol. 3750, pp. 919-926. Springer, Heidelberg (2005)

4. Wang, H., Dong, L., O'Daniel, J., Mohan, R., Garden, A.S., Ang, K.K., Kuban, D.A., Bonnen, M., Chang, J.Y., Cheung, R.: Validation of an accelerated 'demons' algorithm for deformable image registration in radiation therapy. Physics in Medicine and Biology 50, 2887-2905 (2005)

5. Cachier, P., Pennec, X., Ayache, N.: Fast Non Rigid Matching by Gradient Descent: Study and Improvements of the "Demons" Algorithm. Rapports de recherche no 3706, Note 25 (1999)

6. Papademetris, X., Shkarin, P., Staib, L.H., Behar, K.L.: Regional Whole Body Fat Quantification in Mice. In: Christensen, G.E., Sonka, M. (eds.) IPMI 2005. LNCS, vol. 3565, pp. 369-380. Springer, Heidelberg (2005)

7. Li, X., Peterson, T.E., Gore, J.C., Dawant, B.M.: Automatic Registration of Whole Body Serial Micro CT Images with a Combination of Point-based and Intensitybased Registration Techniques. In: ISBI 2006, pp. 454-457 (2006)

8. Li, X., Yankeelov, T.E., Peterson, T.E., Gore, J.C., Dawant, B.M.: Automatic nonrigid Registration of Whole Body CT Mice Images. Medical Physics 35(4), 1507-1520 (2008)

9. Chui, H., Rangarajan, A.: A new point matching algorithm for nonrigid registration. Comput. Vis. Image Underst. 89, 114-141 (2003)

10. Martin-Fernandez, M.A., Munyoz-Moreno, E., Martin, M.: Articulated registration: elastic registration based on a wire-model. In: Proceedings of SPIE 2005, vol. 5747, pp. 182-191 (2005)

11. Rueckert, D., Sonoda, L.I., Hayes, C., Hill, D.L.G., Hawkes, D.J.: Non-rigid registration using free-form deformations: Application to breast MR images. IEEE Transactions on Medical Imaging 18, 712-721 (1999) 